\title{
Los AINEs COX-2 y el misoprostol son las mejores estrategias gastroprotectoras en tratamientos prolongados con AINEs
}

\section{Objetivo}

Valorar la efectividad de 5 estrategias gastroprotectoras para consumidores de antinflamatorios no esteroideos (AINEs): 1) Antagonistas de los receptores H2 + AINE no selectivo; 2) Inhibidor de la bomba de protones (IBP) + AINE no selectivo; 3 ) Misoprostol + AINE no selectivo; 4) AINE COX-2 selectivo (Ej: meloxicam, etodolac) y 5) AINE COX-2 específico (Ej: celecoxib, rofecoxib).

\section{Fuentes de Datos}

Biblioteca Cochrane; MEDLINE, EMBASE, Sistema para Información de Literatura Gris en Europa (SIGLE) hasta el 2002. Se examinaron las referencias y se incluyeron las revisiones sistemáticas identificadas, y se intentó contactar autores.

\section{Selección de estudios}

Dos evaluadores independientes hicieron la selección de ensayos. Los resultados primarios fueron complicaciones gastrointestinales (GI) serias (incluyendo erosiones hemorrágicas, sangrado gastrointestinal alto recurrente, perforación, obstrucción pilórica, melena, y muerte por alguna de estas causas); úlceras sintomáticas; enfermedad cardiovascular (CV) o renal importante; calidad de vida relacionada con la salud; y mortalidad.

\section{Extracción de datos}

La evaluación de los estudios consideró: enmascaramiento de la asignación*, procedimientos de aleatorización, similitud de grupos al inicio, ceguera, y pérdidas en el seguimiento.

\section{Resultados principales}

De los 112 ensayos aleatorizados incluidos con 74.666 participantes se reportaron 138 muertes y 248 eventos GI serios. Como se observa en la tabla, el misoprostol y probablemente los AINEs COX-2 específicos reducen significativamente el riesgo de complicaciones Gl serias. El misoprostol, ambos tipos de AINEs COX-2 y probablemente IBP reducen significativamente el riesgo de úlceras sintomáticas. No se encontró evidencia de efectividad de los antagonistas de receptores $\mathrm{H} 2$ para los resultados primarios (pocos eventos reportados), y tampoco diferencias entre las estrategias en los otros resultados evaluados. Se observó que el misoprostol aumenta los abandonos por efectos GI (RR 1,36; IC95\% 1,3 a 1,5) No se encontró sesgo de publicación*.

Tabla. Resultados primarios para las cinco estrategias del gastroprotectoras.

\begin{tabular}{|c|c|c|c|c|c|}
\hline Resultados pr & \begin{tabular}{|c} 
Antagonistas \\
Hz vs placeho \\
(n=2621)
\end{tabular} & $\begin{array}{c}\text { BBP vs } \\
\text { placebo } \\
(n=1358)\end{array}$ & $\begin{array}{c}\text { Misoprostol } \\
\text { vs placebo } \\
(n=16945)\end{array}$ & \begin{tabular}{|c|} 
COXX-2 \\
selectivos vs \\
no-selectivos \\
(n=20178)
\end{tabular} & \begin{tabular}{|c} 
COXX-2 \\
especifilicos vs \\
noo-selectivos \\
(n=25564)
\end{tabular} \\
\hline & \multicolumn{5}{|c|}{ Riesgo Relativo (IC95\%) } \\
\hline nplicaciones GI serias & $0,33(0,0-8,1)$ & $0,46(0,1-2,9)$ & $0,57(0,4-0,9)^{8}$ & $0,61(0,3-1,1)$ & $0,55-(0,4-0,8)^{t}$ \\
\hline ceras sintomaticas & $1,46(0,1-35,5)$ & $0,09(0,0-0,5)^{t}$ & $0,36(0,2-0,7)^{8}$ & $0,41(0,3-0,7)^{\mathrm{s}}$ & $0,49-(0,4-0,0,6)^{8}$ \\
\hline rentos CV o renales serios & \begin{tabular}{l|l}
5 & $0,53(0,1-3,5)$
\end{tabular} & $0,78(0,1-6,3)$ & $1,78(0,3-12,1)$ & $0,95(0,6-1,7)$ & $1,19-(0,8-1,8)$ \\
\hline talidad & & $0,17(0,0-4,1)$ & $0,89(0,5-1,7)$ & $0,68(0,3-1,6)$ & $1,02-(0,6-1,9)$ \\
\hline
\end{tabular}

\& Significativa gastroprotección $(p<0,05)$ mantenida en el análisis de sensibilidad y sin heterogeneidad.*

\# Significativa gastroprotección $(\boldsymbol{p}<0,05)$ pero no mantenida en el análisis de sensibilidad o con significativa heterogeneidad $(p<0,1)$

\section{Conclusiones}

El Misoprostol y probablemente los AINEs COX-2 específicos reducen el riesgo de complicaciones gastrointestinales serias. $\mathrm{El}$ misoprostol, ambos tipos de AINEs COX-2 y probablemente IBP reducen el riesgo de úlceras sintomáticas pero la calidad general de los datos es pobre. El misoprostol tiene mayores abandonos por efectos GI. Los datos sobre antagonistas H2 e IBP son insuficientes.

Fuente de financiamiento: National Health Service, Reino Unido.

\section{Comentario}

Son conocidos los efectos colaterales GI de los AINEs, desde dispepsias moderadas a muerte por hemorragias digestivas o perforación. Los agentes gastroprotectores son usualmente prescriptos en forma concomitante para prevenirlos. Se compararon la efectividad de éstos vs. placebo y de COX-2 vs. AINEs no selectivos a través de una revisión sistemática metodológicamente rigurosa, que podría ser más definitoria si los eventos importantes se registraran sin excepción en los ensayos. El misoprostol y los COX2 surgen como de utilidad en la reducción de riesgo GI. Se recuerda la clasificación de los AINEs, en convencionales (inhiben la COX-1) selectivos (inhiben la COX-2 pero a mayores dosis también la COX-1), y específicos (inhiben la COX-2). Lamentablemente, los específicos demostraron tener un efecto de clase en el aumento de eventos cardiovasculare $\mathrm{s}^{1-5} \mathrm{y}$ muchos se retiraron del mercado en varios países.
A propósito de datos surgidos del estudio, es importante señalar la dificultad de recabar algunos datos en los trabajos financiados por la industria farmacéutica debido al reporte selectivo, y la necesidad de la puesta en práctica de legislación que obligue a la industria a hacer públicos todos los datos surgidos de ensayos por ella financiados.

\section{Conclusiones del comentador}

El trabajo invita a considerar las potenciales ventajas de los COX2 selectivos, de menor uso pero sin el comprobado riesgo cardiovascular de los específicos; al igual que el misoprostol. De todos modos, un gran ensayo clínico, bien diseñado e independiente debería finalmente definir la mejor estrategia gastroprotectora.

\section{Dr. Ricardo Sarandría [ Médico Becario. Unidad de Medicina Familiar y Preventiva.Hospital Italiano de Buenos Aires. ]}

\section{Dr. Agustín Ciapponi [ Unidad de Medicina Familiar y Preventiva. Hospital Italiano de Buenos Aires. ]}

Sarandría R, Ciapponi A. Los AINEs COX-2 y el misoprostol son las mejores estrategias gastroprotectoras en tratamientos prolongados con AINEs. Evid actual pract ambul 2005;8:70. Lee Hooper, Tamara J Brown, Rachel Elliott et al. The effectiveness of five strategies for the prevention of gastrointestinal toxicity induced by non-steroidal anti-inflammatory drugs: systematic review. BMJ 2004;329:948. PMID: 15475342

\section{Referencias}

1. Furberg CD, Psaty BM, FitzGerald GA. Parecoxib, valdecoxib, and cardiovascular risk. Circulation. 2005;111(3):249.

2. Topol EJ, Falk GW. A coxib a day won't keep the doctor away. Lancet. 2004;364(9435):639-40.

3. Juni P, Nartey L, Reichenbach S, Sterchi R, Dieppe PA, Egger M. Risk of cardiovascular events and rofecoxib: cumulative meta-analysis. Lancet. 2004;364(9450):2021-9.

4. Solomon SD, McMurray JJ, Pfeffer MA, Wittes J, Fowler R, et al. Cardiovascular Risk Associated with Celecoxib in a Clinical Trial for Colorectal Adenoma Prevention. N Engl J Med. 2005 Feb 15 [http://click.nejm.org/ct/click?q=70-3guJldpCVDnHOj8WnRN4sQHjUJlqkEyR]

5. Bresalier RS, Sandler RS, Quan H, Bolognese JA, Oxenius B, Horgan K, et al. Cardiovascular Events Associated with Rofecoxib in a Colorectal Adenoma Chemoprevention Trial. N Engl J Med. 2005 Feb 15 [http://click.nejm.org/ct/click?q=9a-b0CGQTMEGYQww0Jvi79qyecTKzhemFrR] 